\title{
INFECTIOUS DISEASES, NSW - DECEMBER 1997
}

\section{TRENDS}

IT he outbreak of pertussis in NSW has reached epidemic proportions, with 666 cases being reported in November alone. The Chief Health Officer has written to all doctors in the State alerting them to the epidemic, and urging them to:

- consider a diagnosis of pertussis in people with coughing illnesses,

- treat cases of pertussis and their household contacts with erythromycin,

- notify their Public Health Units of suspected cases; and

- vaccinate all children against pertussis, following NHMRC recommendations.

Health care providers should routinely check the vaccination status of all their patients and opportunistically update children's immunisations if they are due. Routine childhood immunisations are free in NSW. In addition, the NSW Health Department has provided funding for acellular pertussis vaccine, available as DTPa (a vaccine containing acellular pertussis vaccine with tetanus and diphtheria vaccines). Immunisation providers can now obtain DTPa free of charge for children due for vaccination at 18 months and 4-5 years of age, as well as for children who have had previous moderate reactions to tripleantigen vaccination. $\mathrm{PHUs}$ will provide further advice on the use of this and other vaccines and the control of pertussis to health care providers and parents.

After an earlier rise in the number of cases, notifications of meningococcal disease further declined through October (Figure A) and November (Table A), with a total of 204 notifications for the year to the end of November.

Laboratory notifications of salmonellosis rose above historical expectations in October, in part because of an increase in Salmonella typhimurium phage type 64 ( 40 cases identified for October). While preliminary investigations have revealed no common source of this infection, further investigations are under way.

\section{OUTBREAK OF GASTROENTERITIS LINKED TO EATING PIPIS}

Julianne Quaine, Ed Kraa, Joe Holloway, Kim White, Rod McCarthy, Valerie Delpech, Marianne Trent and Jeremy McAnulty on behalf of the Public Health Network.

O December 16, 1997 the Food and Nutrition Unit received reports from South Western Sydney, Central Sydney and South Eastern Sydney Public Health Units of five separate groups of people who had been ill after consuming pipis. On December 16 the Food and Nutrition Unit sent an e-mail to all PHUs seeking further reports of gastroenteritis related to pipi consumption. Over the next 13 days details of 56 people with pipi-related illness were received from Central Sydney, South Western Sydney, South Eastern Sydney, Northern Sydney and Northern Rivers. In addition, anecdotal reports of a further 46 people, for whom individual details were not available, were reported.

\section{Pipis}

Pipis (Plebidonax deltoides) are bivalve molluses that live in the intertidal zone of sand on gently sloping ocean beaches, a few centimetres below the surface. In NSW pipis are collected in commercial quantities from the beaches around Ballina, South West Rocks, Forster-Tuncurry and Stockton. Most commercial supplies in NSW come from Ballina, which has an annual collection of 300,000 kilograms. After harvesting, the sand is removed from pipi shells by placing them in salt water for several hours.

\section{Epidemiological investigation}

For this investigation, a case was defined as a person reporting gastrointestinal symptoms of either vomiting or diarrhoea after ingestion of pipis from December 5, 1997. Between December 12 and 16, 56 cases were reported, including 29 females and 27 males. The mean age of cases was 33.9 years (range 9-71 years). Age was unknown for 8 cases. Cases were reported from PHUs in South Western Sydney (18), Northern Sydney (13), Central Sydney (11), Northern Rivers (7) and South Eastern Sydney (7).

Reported symptoms included diarrhoea (56 cases, 100 per cent), abdominal cramping ( 50 cases, 89 per cent), nausea ( 27 cases, 48 per cent), vomiting ( 14 cases, 25 per cent), headache (12 cases, 21 per cent) and fever ( 8 cases, 14 per cent).

Based on the reported time between pipi consumption and illness (reported for 40 cases), the mean incubation period was 16.4 hours (range $2-49$ hours). The mean duration of symptoms (reported for 28 cases) was 49 hours (range 12-84 hours)

Stool samples from three cases were negative for bacteria and (as preliminary results indicate) negative for viruses.

Nine of the cases were among a party of 27 who ate a meal at a restaurant in south-western Sydney. Of the 15 people who completed questionnaires administered by PHU staff, 13 reported eating pipis. Nine of the 13 people ( 69 per cent) who ate pipis at the dinner were ill, compared with no illness in the two people who did not eat pipis at the dinner $(P=0.14)$.

\section{Environmental investigation}

The implicated pipis were traced to a supplier on the NSW north coast. Samples of pipis were tested by the Division of Analytical Laboratories. Standard bacterial plate counts for raw pipis were under $10^{5}$ for all samples, the limit set by Standard D of the Australian Food Standards Code. The level of $E$. coli was above the limit set in Standard D in some raw and cooked samples. Some raw samples, but not all, had levels of Vibrio parahaemolyticus under $10^{4}$. Levels of $V$. parahaemolyticus over $10^{5}$ are associated with illness ${ }^{1}$. Salmonella typhimurium and S. sophia were present in two raw samples and $S$. sophia was present in one raw sample. No enteroviruses or Norwalk viruses were found in any of the pipi samples.

Two frozen samples of implicated pipis collected from cases were tested at the Institute of Environmental Science and Research in Wellington, New Zealand, for the presence of marine biotoxins, specifically diarrhoetic shellfish poisons. Diarrhoetic shellfish poisoning is caused by a group of polyether toxins produced by microalgae of the Dinophysis species ${ }^{2}$. Bivalve shellfish, such as pipis, accumulate the toxin through filter-feeding on microalgae. Results obtained on December 31, 1997 showed that initial ELISA tests for the presence of two specific diarrhoetic shellfish poisons were negative, but a mouse bioassay was positive, 
indicating the presence of a toxin. These results do not rule out diarrhoetic shellfish poisoning as the cause of the illness. Further testing will be undertaken to identify the specific toxin present.

\section{Intervention}

On December 17, 1997 the NSW Department of Health issued a media release warning the public to cook pipis well before eating them. When subsequent cases reported incubation periods as short as 2 hours and consuming pipis that had been cooked for up to 30 minutes, concern that a heat-stable toxin may be responsible for the illness led the Department to issue a second warning, on December 19, that pipis from Northern NSW should not be eaten. The Sydney Fish Markets voluntarily suspended sale of pipis on December 17 and the Ballina Fish Co-operative voluntarily recalled all pipis from sale on December 18. On December 19 the Fisheries Department closed the beaches between the Richmond and Evans rivers on the far north coast to harvesting of bivalve molluscs at least until January 23, 1998.

Pipis implicated in all reported cases are being traced to their source to determine whether the handling processes are implicated as a cause of the contamination. The presence of salmonella in some pipi samples is being investigated. Further testing of pipis is required to determine the specific toxin present and whether the toxin is the cause of the presenting illness.

1. Benenson AS (editor). Control of communicable diseases manual. 16th ed. Washington: American Public Health Association, 1995. 2. Hallegraeff GM. A review of harmful algal blooms and their apparent global increase. Phycologia 1993; 32(2):79-99.

\section{AVIAN INFLUENZA A (H5N1) IN HUMANS, HONG KONG, 1997}

A strain of influenza virus that previously was known to infect only birds has been associated with infection and illness in humans in Hong Kong. The first known human case of influenza type A (H5N1) occurred in a 3-year-old child who died from respiratory failure in May 1997. In Hong Kong the virus initially was identified as influenza type A but the subtype could not be determined using standard reagents. By August the virus was identified as influenza A (H5N1). The possibility of laboratory contamination was excluded. Since this initial case was identified six additional Hong Kong cases have been confirmed and two possible cases were identified to mid-December.

\section{Confirmed cases}

Patient 1: On May 9, 1997 a previously healthy 3-year-old boy developed fever, sore throat and cough. His illness progressed and on May 18 he was admitted to the paediatric intensive care unit. On May 21 the child died from acute respiratory distress secondary to viral pneumonia. Influenza A (H5N1) virus was isolated from a tracheal aspirate collected on May 19. The child may have been exposed to ill chickens before he became ill.

Patient 2: On November 6 a 2-year-old boy with a congenital heart disease developed high fever, cough and sore throat and was admitted to hospital the next day for presumed pneumonia. He had an uneventful recovery and was discharged from the hospital on November 9.

A nasopharyngeal swab yielded influenza $\mathrm{A}$ ( $\mathrm{H} 5 \mathrm{~N} 1)$ virus.
Patient 3: On November 20 a previously healthy 13-year-old girl developed fever, sore throat and cough. She was admitted to hospital on November 26 because of pneumonia. On November 27 she was transferred to the ICU and placed on mechanical ventilation. As of December 17 she remained in hospital. Influenza A (H5N1) virus was isolated from a tracheal aspirate.

Patient 4: On November 24 a previously healthy 54-year-old man developed fever and cough and on November 29 he was admitted to hospital because of pneumonia. His condition deteriorated and he died on December 5 . A bronchoalveolar lavage specimen yielded influenza $\mathrm{A}$ ( $\mathrm{H} 5 \mathrm{~N} 1$ ) virus.

Patient 5: On December 4 a 24-year-old woman developed fever, sore throat, cough and dizziness. Her symptoms worsened and she was admitted to hospital on December 7 . Her condition deteriorated and on December 9 she was transferred to the intensive care unit and placed on mechanical ventilation. Influenza A (H5N1) was isolated from a tracheal aspirate.

Patient 6: On December 7 a 5-year-old girl developed fever, rhinitis, cough, sore throat and vomiting. As of December 17 she remained in hospital in satisfactory and stable condition. A nasopharyngeal aspirate yielded influenza A (H5N1).

Patient 7: On December 12 a 2-year-old boy developed fever and was admitted to hospital in good condition. The child is a cousin of patient 6 , who frequently visited him and his family at their home. A culture from the child was positive for influenza A (H5N1) virus.

\section{Possible cases}

On November 24 a previously healthy 37 -year-old man was hospitalised because of pneumonia; the onset of the illness was on November 17. He recovered and was discharged. Preliminary results of serologic tests suggest infection with influenza A (H5N1); results of a neutralisation assay (which is required to confirm infection) are pending. The other possible case is the 3 -year-old sister of patient 7 and cousin of patient 6 . She lived in the same apartment as patient 7 and had the onset of fever on December 13 and was admitted to hospital in good condition. Preliminary laboratory results were positive for influenza A (H5N1) virus; confirmation of these results by virus isolation is pending.

\section{Ongoing investigation}

The primary objectives of the ongoing investigation are to detect and investigate new cases and to identify potential sources, including whether and to what extent infection is being transmitted from person to person, birds to humans, or both. Blood specimens for measurement of antibody against influenza $\mathrm{A}$ (H5N1) and information concerning respiratory illness, exposure to birds, the type and degree of exposure to cases, and other relevant information are being collected from people who had contact with cases and from control groups that did not have contact with cases.

Patients 1 to 6 lived in different parts of Hong Kong, had no contact with each other and had no apparent common exposures. Patients 6 and 7 and the 3-year-old girl who is a possible case have all had contact with each other and common exposures. Influenza A (H5N1) viruses isolated from these patients are being fully characterised both 
antigenically and genetically by the United States Centers for Disease Control and Prevention.

Surveillance for influenza has been intensified in Hong Kong and Guangdong Province, China, following the identification of the first human case, mostly through outpatient facilities and hospitals. Surveillance among poultry in Hong Kong indicates continued circulation of A (H5N1) viruses since March, when outbreaks on poultry farms were first detected.

\section{Comment}

The cases described in this report represent the first documented human infections with avian influenza $\mathrm{A}$ (H5N1) virus. One of the most important aspects of the investigation is to determine the source of infection and mode of transmission. This effort is complicated by the high prevalence of exposure to live poultry among residents of Hong Kong.

Although the spectrum of illness caused by human influenza virus infection can range from asymptomatic to fatal, most human influenza infections cause acute febrile respiratory illnesses that resolve without complications. Many of the cases of human infection with type A (H5N1) identified in Hong Kong have been unusually severe. However, because influenza surveillance in Hong Kong has been conducted primarily in hospitals, milder cases may not have been recognised, and the severity of infections identified to date may not be representative of the spectrum of illness caused by A (H5N1) infection in humans.

Infection with this influenza strain, which is new to humans, prompts consideration about whether this virus has the potential to spread globally and cause a pandemic. For an influenza pandemic to occur, a novel human influenza strain against which all or most of the human population has no antibody must be capable of sustained person-to-person transmission, causing widespread illness ${ }^{1}$. As of December 17 acute respiratory illness among the population of Hong Kong apparently had not increased.

Although the potential for widespread transmission of this strain is unknown, as a precautionary measure laboratory studies have been initiated to identify a candidate A (H5N1) vaccine strain. There are no plans for commercial vaccine production. Two antiviral drugs, amantadine and rimantadine, inhibit replication of virtually all naturally occurring human and animal strains of influenza type A and therefore can be useful for prophylaxis and treatment of influenza A infections ${ }^{24}$. Influenza A viruses resistant to amantadine and rimantadine can emerge during treatment, but drug-resistant influenza viruses have only rarely been isolated from specimens collected as part of routine influenza surveillance ${ }^{5,6}$. Influenza A (H5N1) isolates from Hong Kong that have been tested are sensitive to amantadine and rimantadine.

People considering travel to Hong Kong should consider that:

- the number of clinical cases of influenza A (H5N1) identified to date is small, despite the intensive surveillance that has been conducted among the 6.5 million residents of Hong Kong; and there has been no detected increase in the incidence of acute respiratory illness among residents of Hong Kong. However, the risk for infection to people living in, or visiting, Hong Kong cannot be determined with certainty, and the risk may change over time. Although no human influenza A (H5N1) infections have been identified outside Hong Kong, worldwide surveillance for influenza is critical to monitoring the circulation of various influenza strains. Human influenza types A (H3N2), A (H1N1) and B continue to circulate worldwide ${ }^{7.8}$.

\section{Avian influenza (H7N4), Tamworth}

The avian influenza virus in Hong Kong is an entirely different strain from the one that affected chicken and other bird flocks in the Tamworth area in November and December 1997. An increased mortality rate (up to 96 per cent) was noted on a Tamworth chicken farm in mid-November. Viral studies indicated the cause of that outbreak was avian influenza A (H7N4). This virus has not been shown to affect people or mammals or the quality of poultry meat or eggs, but is important to the agriculture industry because of its potential economic effect. NSW Agriculture reports that this is the fifth outbreak of avian influenza in Australia in the past 20 years. This and all previous outbreaks were successfully eradicated. The cause of such outbreaks is hypothesised to be contamination of drinking water by wild birds (especially water fowl).

\section{Implications of Hong Kong avian influenza for NSW} This report has raised concern about the possible emergence of a new strain of influenza. New strains of influenza were responsible for devastating epidemics in 1918-19 (more than 20 million deaths), 1957 (70,000 deaths in the United States alone), and 1968-69 (33,000 US deaths). However, it is quite possible that such avian strains may occasionally infect humans (who act as a dead-end host), but be identified only because of the intense surveillance for emerging influenza strains that has been going on in Hong Kong and southern China as part of a World Health Organisation influenza pandemic early-warning system.

The NSW Health Department is working closely with the Commonwealth and other State and Territory Health Departments to develop enhanced influenza surveillance and control measures. A group of local public health, laboratory, primary care and infectious disease experts will be convened early in 1998 to provide advice as events unfold.

Adapted from: CDC. Isolation of Avian Influenza A (H5N1) Viruses from humans - Hong Kong, May-December 1997. MMWR 1997; 46:1204-7.

1. Cox N, Patriarca P. Influenza pandemic preparedness plan for the United States. I Infect Dis 1997; 176(suppl 1):S4-S7.

2. Douglas RG. Drug therapy: prophylaxis and treatment of influenza N Engl J Med 1990; 322:443-50.

3. Tominack RL, Hayden FG. Rimantadine hydrochloride and amantadine hydrochloride use in influenza A virus infections. Infect Dis Clin North Am 1987; 1:459-78.

4. Hayden FG. Antivirals for pandemic influenza. J Infect Dis 1997 ; 176(suppl 1):S56-S61.

5. Belshe RB, Burk B, Newman F, Cerruti RL, Sim IS. Resistance of influenza $A$ virus to amantadine and rimantadine: results of one decade of surveillance. J Infect Dis 1989; 159:430-5.

6. Ziegler T, Hemphill M, Zeigler ML, Klimov A, Cox N. Rimantadine resistance of influenza $A$ viruses: an international surveillance.

Presented at the 7th ISAR Conference, Charleston, South Carolina, March 1994

7. CDC. Update: influenza activity - worldwide, March-August 1997. MMWR 1997; 46:815-8.

8. CDC. Update: influenza activity - United States, 1997-98 season. MMWR 1997; 46:1192-4. 


\section{FIGURE 8}

REPORTS OF SELECTED INFECTIOUS DISEASES, NSW, 12 MONTHS TO OCTOBER 1997, BY MONTH OF ONSET (WITH HISTORICAL COMPARISON)
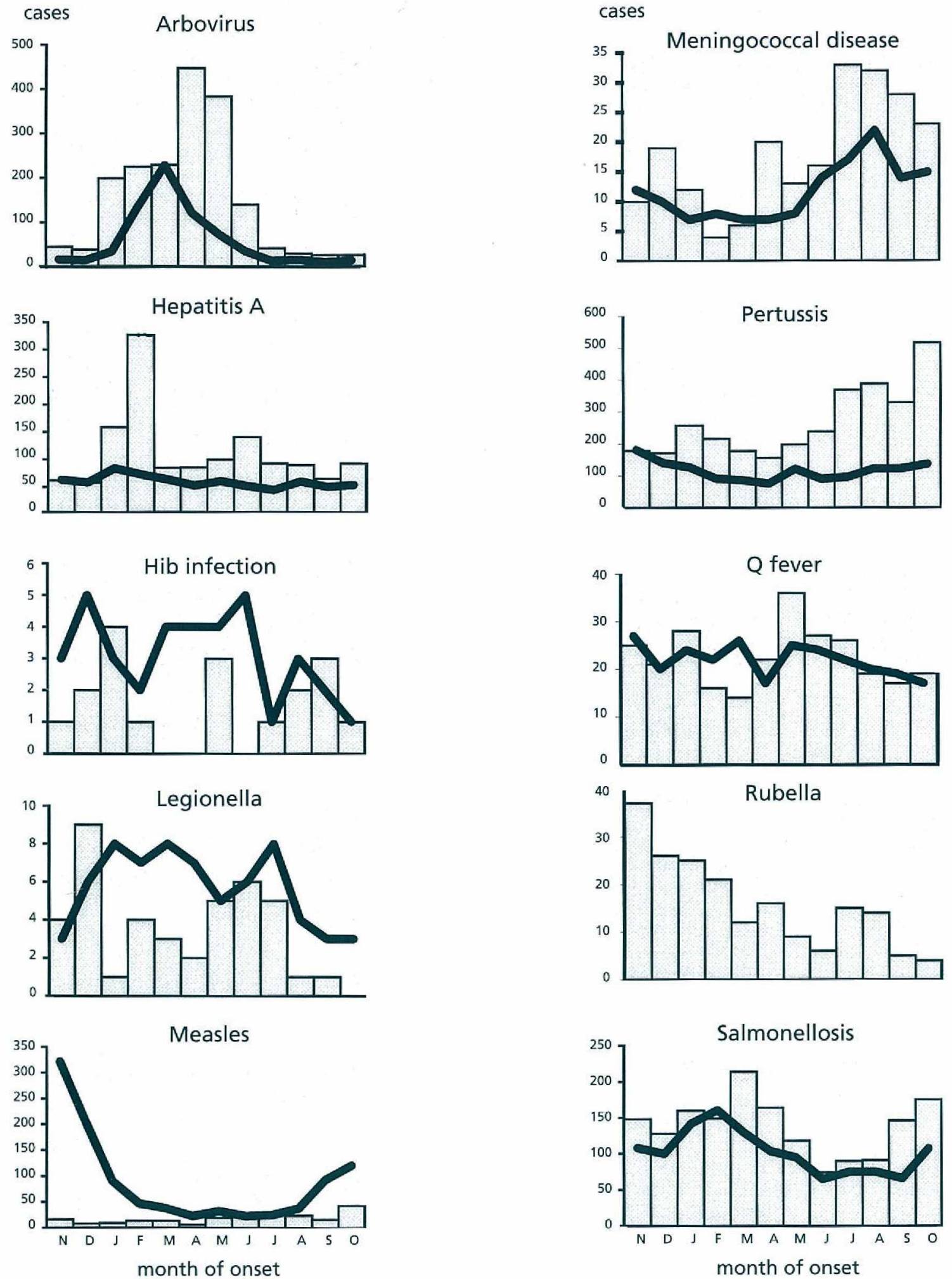

Because of data collation problems, historical rubella figures are unavailable. 
INFECTIOUS DISEASE NOTIFICATIONS FOR NSW RECEIVED IN NOVEMBER 1997 BY AREA HEALTH SERVICES

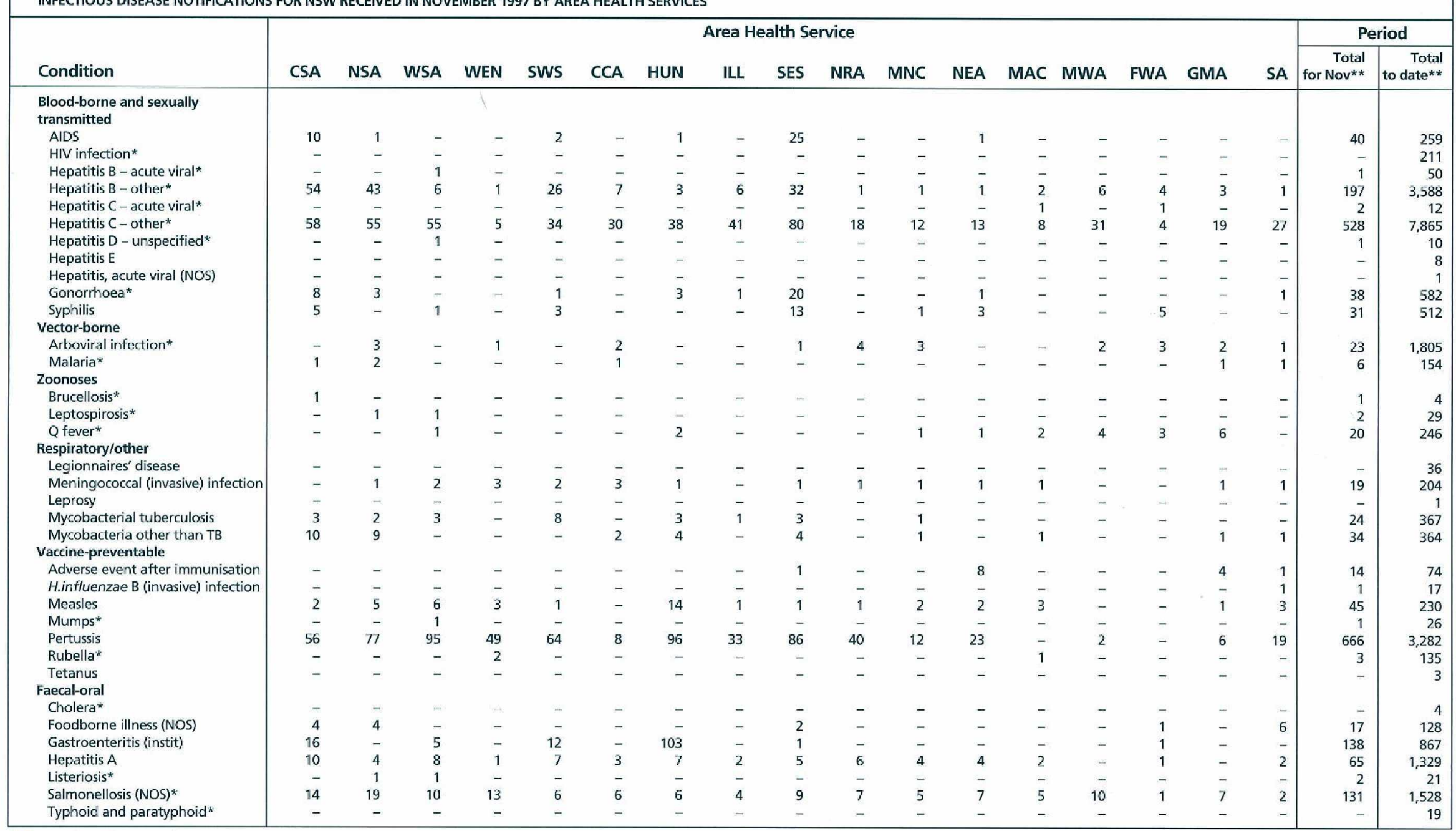

* lab-confirmed cases only
$* *$ includes cases with unknown postcode

Abbreviations used in this Bulletin:

CSA Central Sydney Health Area, SES South Eastern Sydney Health Area, SWS South Western Sydney Health Area, WSA Western Sydney Health Area, WEN Wentworth Health Area,

NSA Northern Sydney Health Area, CCA Central Coast Health Area, ILL Illawarra Health Area, HUN Hunter Health Area, NRA Northern Rivers Health Area, MNC Mid North Coast Health Area, NEA New England Health Area, MAC Macquarie Health Area, MWA Mid West Health Area, FWA Far West Health Area, GMA Greater Murray Health Area, SA Southern Health Area, OTH Interstate/Overseas, U/K Unknown, NOS Not Otherwise Stated. 